\title{
9. Reflection in self-organised systems
}

\author{
Maureen Lynch, School of Information Systems, University \\ of South Australia \\ Carmen Johan, School of Information Systems, University \\ of South Australia
}

\begin{abstract}
Organisations operating in a dynamic environment need to be able to detect and respond to changes both internal and external. One effective decision making approach is selforganisation, which is appropriate where there is a state of constant awareness of the working environment and flexibility so that decision-making groups are formed spontaneously to solve problems and incorporate changes. Decision-making from this perspective is done on three levels: individual, group or cluster, and organisational. With each level, it is imperative that the situation is fully understood, alternative solutions are considered and compared with an ideal, and consequences are taken into account. The authors argue that this model of thinking and decision making is that recommended by Dewey - reflection. The evidence used in this argument comes from two bodies of knowledge, the reflection and self-organised systems literature. The paper describes the process of reflection on the three levels of organisational decision making and concludes that one of the most effective ways for organisations to optimise operations in a dynamic environment is to be open to self-organisation and reflective thinking.
\end{abstract}

\section{Introduction}

The complexity of information systems and technological changes confronting most organisations today means there is an increased urgency for them to be able to reflect and adapt. The aim of this paper is to explore the importance of reflection for successful problem solving in self-organised social human systems that face this urgency. Organisations are constantly exposed to new market opportunities and competitive dynamics, demanding that they learn quickly when there is new information provided by, and new opportunities caused by, changes in the external environment.

The increase in interconnectivity and the ubiquity of information systems across the globe is causing the competitive environment to become more complex and self-organising. In this paper, the authors highlight that, in order to operate effectively at the edge of these complexities, reflection at three levels of decision making (i.e. individual, cluster, and organisational) becomes not only necessary but fundamental. An iterative reflection process throughout problem solving, where the 'theory of action' of both individuals and the organisation are questioned and matched against an ideal outcome, allows more informed decisions to be made. This continuous cycle of reflection leads to spontaneous self-organising behaviours. Behaviours are self-organising when knowledge networks and communication channels are freely developed in organisations, even if this involves individuals (actors) crossing the organisational boundaries created by formal hierarchical structures. 


\section{Reflection}

This paper refers to Dewey's (1997) method of thinking and problem solving - reflection that involves the spontaneous creation of a belief (Dewey, 1997), conjecture (Popper, 1969) or intuition (Georgiou, 2001) from one's consciousness and past experience, contemplating and pondering these thoughts, comparing them with an ideal, and finally challenging them in an attempt to substantiate or invalidate them.

Dewey (1997) expresses this reflection succinctly:

Active, persistent and careful consideration of any belief or supposed form of knowledge in the light of the grounds that support it, and the further conclusions to which it tends, constitutes reflective thought. (p6)

The continuous cycle of experiencing, reflecting, forming possible solutions, and testing is illustrated in Kolb's model (see Figure 9.1) as depicted in Graeff (1997). Kolb (1984) argues that, rather than viewing experiential learning as a closed cycle, it should be seen as a spiral 'filling each episode of experience with the potential for movement, from blind impulse to a life of choice and purpose.'

\section{Figure 9.1. Kolb's model of reflection.}

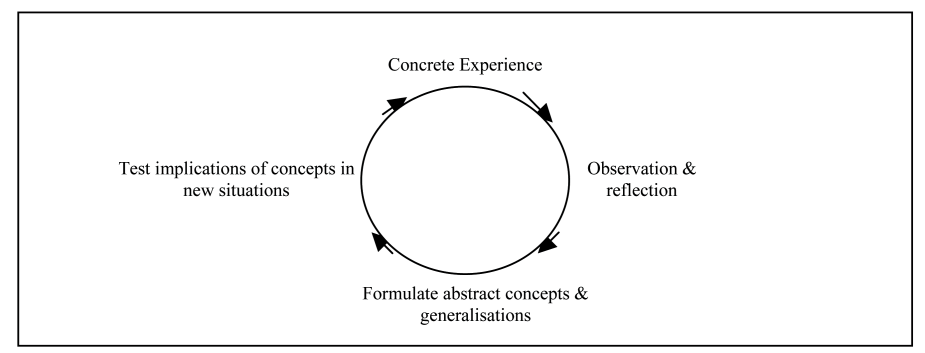

Reflective learning 'engages the person at the edge of their knowledge, their sense of self and the world as experienced by them' (Brockbank and McGill, 1998) where their ideas, beliefs or knowledge are challenged.

\section{Reflection types}

\section{Simple reflection}

Simple or single-loop reflection refers to increasing efficiency of an objective; 'Are we doing things right?' (Flood and Romm, 1996) - it is task oriented and is about the design of the process to retain reliability. It is simple reflection that may challenge assumptions and strategies to alter the plan of action but always in ways that leave the values of a theory of action unchanged' (Brockbank and McGill, 1998). Courtney et al. (1998) describe this as low-level as it involves keeping to a set of rules and is simply error correction. This reflection and learning are viewed as valuable for day-to-day activities and are necessary for progress to be made within the established frameworks (Brockbank and McGill, 1998). To illustrate this sort of reflection, Dooley (1999) uses the example of a buggy whip manufacturer in the early 21 st century improving the manufacturing process in order to make finer buggy whips. The manufacturer does not look beyond his immediate task to take in the strategic perspective of, for example, the long term viability of buggy whip manufacturing. Similarly, an organisation may upgrade a transaction processing system to handle processes more efficiently without the effectiveness or even necessity of the processes being examined. 


\section{Double-loop reflection}

Double-loop reflection is described by Argyris and Schon (1996), who use the term 'double loop learning', and Courtney et al. (1998) as a higher level of reflection than single-loop reflection - it incorporates the first loop (that centres on finding the best means of achieving an end) together with a second loop. This second loop centres on the examination and reflection of the theory or perspective in use. It is recognised that the action and consequences striven for in the first loop may not be valid - that there may be different perspectives regarding what the outcome should be and therefore assumptions, premises and context are questioned. Consequently, double-loop learning asks, 'Are we doing things right AND are we doing the right things' (Flood and Romm, 1996). While it is advised that the 'gate' into the second loop should be used frequently, it cannot be sustained constantly (Brockbank and McGill, 1998) as the required paradigmatic shift that the second loop requires is often disruptive to everyday activities. Dooley (1999) gives as an example of double-loop learning when, in the 1980s, Royal Dutch Shell delayed its plans for acquisition of oil fields when it foresaw the drop in oil prices and the demise of the Soviet Union. It examined what it was doing, as well as how it was doing it. See Figure 9.2 for an illustration of the combination of the two loops to form double-loop learning as depicted in Encyclopedia/Forum (2004).

Figure 9.2. Schon's double-loop reflection.

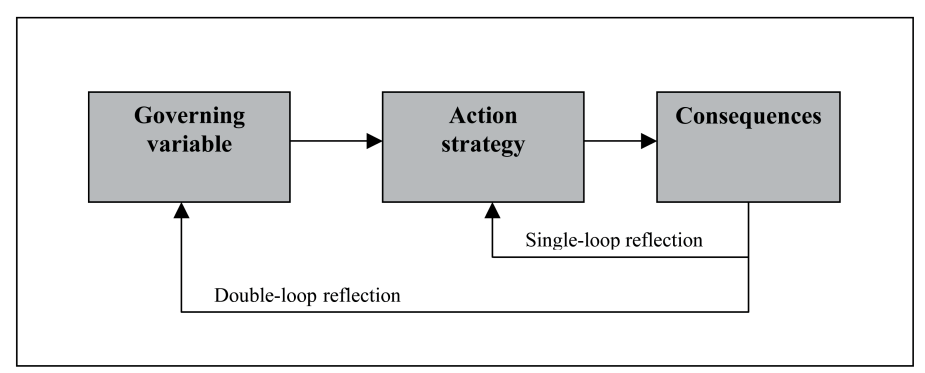

\section{Reflecting against ideal}

Morris and Moore (2000) maintain that research has shown that 'the way individuals make sense of experienced outcomes is greatly determined by thoughts of what could have been, by comparisons of actual outcomes to counterfactual alternatives'. When preparing for an action, 'upward counterfactual comparisons' as anticipatory reflection lead to enhanced outcomes. Therefore, reflection against an ideal, or counterfactual alternatives, when anticipating or reviewing action has been shown to improve the outcome of the process.

There is a double movement in all reflection: a movement from the given partial and confused data to a suggested comprehensive (or inclusive) entire situation; and back from this suggested whole - which, as suggested, is a meaning, and idea - to the particular facts, so as to connect these with one another and with additional facts to which the suggestion has directed attention (Dewey, 1997, p. 80).

Dewey (1997, p. 12) warns that it is impossible to reflect against an ideal if one has not had experience in a similar situation: 'But unless there has been experience in some degree analogous, which may now be represented in imagination, confusion remains mere confusion. There is nothing upon which to draw in order to clarify it'. 
Information systems foundations

\section{Learning from negative feedback}

'It is a theory of experience that assigns to our observations the equally modest and almost equally important role of tests which may help us in the discovery of our mistakes. Though it stresses our fallibility it does not resign itself to scepticism, for it also stresses the fact that knowledge can grow and that science can progress - just because we can learn from our mistakes.' Popper (1969, p. vii)

Negative feedback in this paper is approached from the self-organisation perspective. The concept of reflection involves criticising one's conjectures, ideas, theories or past actions with a view to invalidating them or, in the case of action, to improve performance by learning from mistakes in previous action. Jung (1950), as cited in Fortune and Peters (1997), asserts that 'little or nothing is learnt from successes ... while failures are, on the other hand, priceless experiences in that they not only open up the way to a deeper truth, but force (one) to change views and methods'. Popper (1969) concurs, maintaining that mistakes indicate a gap in knowledge and that learning occurs when those mistakes are rectified.

Fortune and Peters (1997) stress that the main deterrent to learning from failure is 'postevent rationalisation' where frequently the desire to discover the reasons for failure cause investigations to be carried out hastily; this leads to a false picture of the failure, its cause and the context in which it occurred. They cite the cases of the Bhopal chemical disaster and the British European Trident Papa India air crash as examples of hastily formed first impressions that led to completely erroneous findings. The writers advocate using the Systems Failure Method whose goal is 'a systemic interpretation of a failure and its context' (Fortune and Peters, 1997, p. 64) to learn from any failure or mistake. This applies systems thinking concepts to reflection - the focus of reflection is viewed as a 'system' and, when reflection is taking place, the boundary, environment, purpose and different perspectives are taken into account to describe the system in which the failure occurred. An idealised model of the system is designed, and then this and the failed system are compared. This comparison is used to identify or explain the causes of failure. The knowledge created from this reflection is used to recommend actions to improve the situation.

\section{Reflective culture}

This section will argue that the reflective culture of the individual and organisation is critical for effective reflective learning to take place (Schon, 1995; Mathiassen and Sandeep, 2002; Seibert, 1999; Raelin, 2001; Ayas and Zeniuk, 2001; Brockbank and McGill, 1998). Individual reflective culture is defined as 'the act of developing the intellectual and moral faculties' to reflect (Merriam-Webster). It can be difficult for an individual to acquire the skills of reflective learning (Alvesson and Skolberg, 2000) as these skills do not occur naturally. Accordingly, opportunities and education need to be provided to stimulate the practice and develop the individual's culture (Raelin, 2001). Ayas and Zeniuk (2001) agree that a reflective culture based on the search to improve knowledge by questioning one's assumptions and understandings, and the consequences of one's actions, starts at an individual level and can be practiced in the workplace in such situations as project development.

The reflective culture of an organisation is defined as: 'The set of shared attitudes, values, goals and practices that characterises a company or corporation' (Merriam-Webster). If reflective learning is to take place at an organisational level, the culture of the organisation needs to be sympathetic to reflection. Raelin $(2001$, p. 13) maintains that an individual's background has a significant effect on the propensity to reflect with others; some mar- 
ginalised groups have difficulty in developing trust and a sense of security so reflective learning can be threatening unless the environment intellectually and emotionally supports individuals in their learning and development' by allowing them to challenge ideas and practices without the threat of reprisal. It is a common trend in the busy workplace for reflection-in-action and reflection-on-action to be discouraged and resisted due to time pressure (Brockbank and McGill, 1998; Ayas and Zeniuk, 2001), and any type of learning to be deferred to the future. Project work, in particular, allows opportunities for learning, but evidently these opportunities are rarely explored. Sharing of lessons learnt from experiences, successes and problems does not happen in many organisations (Mathiassen and Purao, 2002).

Mathiassen and Purao (2002) suggest that what organisations strive for is a culture that instigates the evolution of a 'community-of-practice'. This is where colleagues collaborate and share work experiences with the intention of combining individual learning in a localised context to allow the creation of knowledge that can be generalised to other organisational contexts and perhaps lead to changes in practices, modifications to design, etc. Communities-of-practice cannot be designed - they emerge as colleagues collaborate to solve problems together. Mathiassen (2002) explains 'membership [of communitiesof-practice] is informal and based on participation in diagnosing situations and telling stories about them. The resulting communities are fluid rather than bounded, evolve rather than being designed and typically cross the formal boundaries of an organisation.'

Successful reflection, therefore, is dependant on culture at both an individual and organisational level. On an individual level, one needs to be in a philosophical position to feel comfortable about questioning long standing assumptions, values and understandings, and with using the reflection process to reach new knowledge. Individual reflective culture can determine organisational reflective culture but organisational culture can also dictate individual behaviour (Ayas and Zeniuk, 2001). If organisational learning is to take place, there needs to be an environment in which individuals feel psychologically safe to challenge organisational norms and practices so double-loop and triple-loop learning can take place.

\section{Self-organisation}

Technological systems become organised by commands from outside, as when human intentions lead to the building of structures or machines. But many natural systems become structured by their own internal processes: these are the self-organising systems, and the emergence of order within them is a complex phenomenon that intrigues scientists from many disciplines (Yates et al., 1987, cited in Camazine et al., 2001).

Self-organisation is a process that is set in motion when, confronted with change, components of a system (e.g. individuals, organisms, elements) spontaneously form patterns and structures in order to target their goals: problem-solving. One way of understanding self-organisation is to contemplate a common purpose or problem, initiating a strong relocation of energy and actions within a system, which leads to the formation of complex webs from elements that are sparsely coupled in order to achieve a common purpose. Following are four definitions that summarise the view of self-organisation as it is being used in this paper:

Self-organisation refers to a broad range of pattern-formation processes in both physical and biological systems, such as sand grains assembling into rippled dunes, chemical reactants forming swirling spirals, cells making up highly organised tissues, and fish joining together in schools (Camazine et al., 2001). 
Self-organisation literally denotes the process whereby a group of people organise themselves in pursuit of a common cause (Humphrey, 2000).

[Self-organisation is] the flexibility of a system to deal successfully with variety in transactions with the environment (Molleman, 1998).

Self-organisation is an emergence of order to deal with social complex systems (Yates et al., 1987).

The term, pattern, as used in this paper, denotes a particular organised assembly of elements taking place in a specific space and time. Based on local information, a system's components interact to create a pattern. As such, a pattern's formation is built with no external direct influence; that is, with no global information or directions from a leader. Patterns, from Kauffman's (1995) point of view, are the creation and physical representation of order; while order is then accounted to the theory of emergence as one of its creations. He argues that order arises naturally as an expression of the self-organisation that abounds in very complex networks. Similarly, in social systems and organisations, the formation of clusters by people themselves pursuing a common cause is a clear example of self-organising patterns.

\section{Constructs of self-organisation}

Rycroft and Kash (2004) state that the world is full of self-organising systems that form structures and processes in response to their own internal logic. A review of the literature (see, for example, Kauffman, 1993; Comfort, 1994; Hudson, 2000; Camazine et al., 2001) suggests that these types of systems have three basic components. Holland and Melhuish (1999) more specifically point out there are three distinctive signatures that complement the four basic characteristics of self-organisation. The three signatures are:

1. the creation of spatio-temporal structures;

2. the possible attainability of different stable states (multiple stable interactions within a system or parallel-processing systems, where various components perform various functions concurrently in order to achieve a desired outcome); and

3. the capacity for adaptation to the prevailing environment.

\section{Spatio-temporal structures}

Self-organisation is a problem solving process whereby components or elements at one particular level interact in order to create structures at a higher level, which may combine again to create even higher level structures. The structures emerging from these repeated interactions develop patterns that are then recognised as self-organisation. Jointly with the environment's space and time, they define the first signature of self-organisation: spatio-temporal structures. An example commonly cited is the spatio-temporal patterns of army ant raids. The coordinated, functional structure of their movement, which spontaneously forms a higher order structure, occurs with minimum external interference.

\section{Multiple interactions}

Self-organisation takes place in systems with multiple active interactions among many actors. Because there are many, often identical, actors there is no requirement for a single actor (e.g. a leader) to carry out a series of connected sequences of movements. For example, referring to an army of ants foraging without recruitment, the rules of thumb ${ }^{1}$ are just simple cues that alone will ensure that a complete sequence of actions is executed, 
even though an action or movement may be performed by a different ant from the one initially involved in it. The execution of these rules, in this case, is conducted by the flow (cue) of an ant's pheromone. Multiple stable interactions arise when other ants interact with this cue and create new stimuli for further interactions to occur.

Ants leave their nest in order to find food. Once found, they load up and return to the nest leaving a trail of pheromone. For simplicity, assume that ants 'would raise an alarm for other ants to follow'; while this is a multiple interactive process, it could also be a parallel-process as other ants from the nest may find other food sources and also raise an alarm. However, as Holland and Melhuish (1999, p. 4) assert, ' ... if there are many locations with such cues, the subtask ${ }^{2}$ will be performed faster at the location that has greatest numbers of agents present ${ }^{\prime}$ due to the higher interaction rate and stability of the process.

\section{Adaptation}

Prigogine (1996, p. 711) argues that '... self-organising systems allow adaptation to the prevailing environment'. Comfort (1994, p. 3) explains that:

... self-organisation recognises that individual choices, communicated across organisational frameworks, affect the operation of the wider system [and] in this respect, voluntary selection allows individuals operating within organisational systems to cluster around points of energy that they find more attractive, creating a 'peak' of energy distribution over repeated interactions and aligning other members to that point in a 'basin' of attraction.

This explains why ants perform faster at the location that has greatest numbers of ants present: they cluster around the point of more attractive energy, adapting to environmental conditions (Kauffman, 1993; 1995). This actually becomes a powerful mechanism when coupled with one of the formal characteristics of self-organisation: positive feedback.

In summary, self-organisation is the result of utilising the system's capacity for patterns and structure formation; processing communication and multiple interactions by choices or cues; and the mutual adjustment in behaviour based on a shared goal among actors of a given system and environmental conditions. Based on these signatures, we can now start to identify the main four characteristics of that system.

\section{Positive and negative feedback}

While most self-organising systems use positive feedback, for such systems both negative and positive feedback are indispensable. Camazine, et al. (2001) point out that negative feedback often takes the form of regulation, competition, reduction or saturation. Continuing with the social insect analogy: in the ants' nest negative feedback dominates when there is competition among food sources, the food source is fully consumed, too many ants are feeding from a food source, there are not enough food sources in a particular area, lack of space or any other similar event that overtakes the positive feedback processes of the ants' nest. Consequently, the ants are forced to hunt for other food sources and commence the feeding cycle again. A different example used in the biology literature is the case of pillar formation in termite nests (e.g. Franks and Deneubourg, 1997; Camazine et al., 2001). In this event, negative feedback takes over when there is no more material in the area close to the formation of these types of pillars. It has also been observed that there seems to be a certain type of competition among termites

${ }^{2}$ This is the sequence of movements making up a task; in this case: search, food and nest. 
building other pillars in the same area. This pattern of competition is recognised as negative feedback.

Positive feedback coupled with negative feedback provides a powerful mechanism for creating and balancing structures and patterns in many physical and biological systems. Kauffman (1995) points out that feedback and its consequences also apply to organisations - driven by simple behavioural rules, actions and activities. Examples include attraction, aggregation, self-enhancement, clustering and amplification, and they lead to the processes of self-organisation within an organisational system.

\section{Information, communication and cooperation}

Another characteristic of self-organisation is the reliance of organisational processes on multiple interaction and passing of information among individuals. In fact, as Fuchs (2003) points out, ' $\ldots$ all self-organising systems are information-generating systems' and thus, '... information is a relationship that exists as a relationship between specific organisational units of matter'. Systems use communication to process meaning and perform internal and external operations, but it appears that it is the search for information that triggers the emergence of internal order.

Kauffman $(1993$; 1995) asserts that the patterns of communication of information in biological systems are characterised by cues and signals. It is the same for human systems. As Mingers (1997) describes it, the communication of information does not necessarily have to be characterised by language; symbolic interaction via cues or signs alone can generate the information transfer between individuals in a system.

Vanderstraeten (2000) asserts that the identity of information is established in the communication process. But what is the purpose of information? Based on systems theory (e.g. Checkland, 1981) information can be considered as the objective relationship between the elements inside the system's structure and the environment of the system. This basically means that the purpose of information is to establish the relationship of reflection between a system and its environment. This interaction causes structural changes, which result in order, to emerge in the system. It is important to highlight that a system's environment also refers to the surrounding self-organised systems - or neighbours - from which information can be gathered.

Camazine, et al. (2001) assert that, in a good number of the cases, the most important information comes directly from an individual's closest neighbours. So, it could be argued that information is a result of a cooperative process with an individual's neighbours, from which coordination emerges. Fuchs (2003) points out that a detailed study of nature shows that cooperation within animal species and biological organisms is a main aspect of self-organisation. Human beings differ from animals in various ways but cooperation is, of course, also necessary for the existence of social systems. Even competitive situations that create negative feedback (e.g. competition among termites when building pillars) can still be considered cooperative processes that generate information (i.e. termites' interaction) and cause a new order to emerge (i.e. new pillar constructions).

Fuchs (2003) asserts that, in a communication process, a portion of subjective, systemic information (cognition) is conveyed; hence cognition becomes the third aspect of information-generation in self-organising systems. He reiterates that ' $\ldots$ information in selforganising systems has cognitive (subjective), communicative (new subjective information [= structures] emerges in systems due to interaction) and cooperative aspects (interaction results in synergies that cause the emergence of new, objectified information in the shared environment of the involved systems)'. These general aspects can be found in 
biological, physical and social self-organising systems. However, there are qualities unique to each of these systems and their correspondent environments.

\section{Stigmergy}

The term 'stigmergy' was originally proposed in 1959 by the French scientist, Grasse, in his study of social insects, and more specifically, while observing termite building behaviour. Grasse's stigmergy definition, as translated by Holland and Melhuish (1999, p. 2), indicates that:

'... the coordination of tasks and the regulation of constructions does not depend directly on the workers, but on the constructions themselves. The worker does not direct his work, but is guided by it. It is to this special form of stimulation that we give the name Stigmergy (stigma, wound from a pointed object; ergon, work, product of labour = stimulating product of labor)'

This means that stigmergy describes the influence that information, derived from the local environmental effects of the activities of previous individuals, has on the current individuals' behaviour.

Camazine, et al.(2001) refer to stigmergy as the process of information gathering from work in progress. In other words, working stimulus comes from the information gathered when individuals interact with environmental effects rather than from fellow workers. This is a further step in communication and cooperation among individuals of a system. As Camazine, et al.(2001, p. 24) describe it, in continuing with the social insect analogy:

'... instead of coordination through direct communication among nestmates, each individual can adjust its building behaviour to fit with that of its nestmates through the medium of the work in progress'.

Stigmergy appears to be an important mechanism that assists a system to structure itself through the collective behaviour of individuals within the system's environment. An individual could move through the environment, gathering or emitting information, but it can also interact with the environment. Both actions could be considered stigmergy.

Self-organisation is made possible by the coordination of activities that over time and space creates a pattern of construction. Stigmergy is effective in coordinating these construction activities and also in mediating interactions among workers through the environment. For that reason, it is an important component of self-organisation. However, it appears that stigmergy is not a complete explanation of such construction activities since there is no explanation of how construction ends, nor how errors made during construction are amended.

Stigmergy can explain the simpler aspects of transfer of information among individuals, where each individual needs to determine what to do and where a direct line of information from one individual to another individual does not exist; transfer instead occurs through the stimulus of previous individuals' information and construction activities embedded in the environment. Figure 9.3 summarises this process. Here the work previously accomplished by one or more individuals is imprinted in the environment as a cue and stimulus for other individuals. Thus individuals can interact socially by indirect transfer of information encountered in the environment through constructs made by others of their kind. 
Figure 9.3. Stigmergy - information flow.

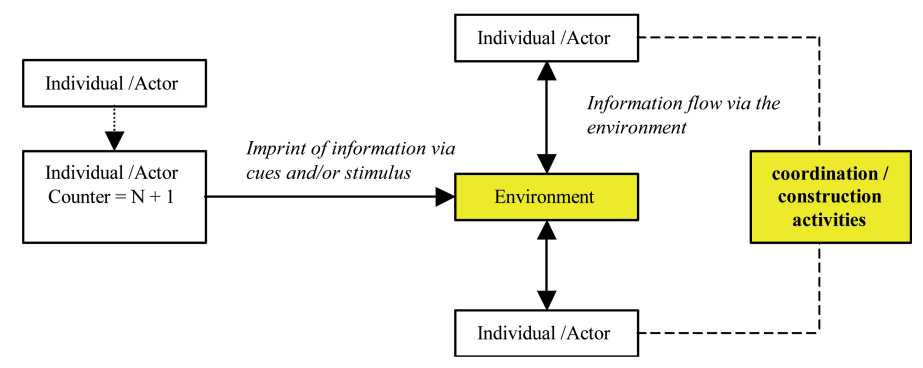

\section{Decentralised control}

Camazine, et al. (2001) refer to decentralised control as another important concept underpinning self-organising systems. As with stigmergy, this concept addresses the flow of information within a system. In decentralised control mechanisms, the information gathered and shared by individuals does not follow a hierarchy of control. In fact, quite the opposite occurs; each individual gathers and acts on information independently. As a result, a natural coordination process of information shared and tasking among individuals takes place without relying on instructions from a leader. Each individual selects essential information for decision-making. This information often originates from the interaction among individual members of the system. This decentralisation of information flow provides the basis for multiple interactions among the components of a self-organised system, making these systems dynamic.

The dynamics of the system (i.e. the interactions, cues and stimuli among individuals, and the environment) are a clear indication of the relationship between stigmergy, decentralised control and self-organisation. As Camazine, et al. (2001, p. 61) point out:

In a decentralised system, each individual gathers information on its own and decides for itself what to do. Stigmergy is one means of information flow within a decentralised system that involves gathering information from the shared environment ... These decentralised paths of information flow provide essential means of interaction among the components in a self-organising system.

Moreover, the multiple interactions are in themselves dynamic processes of pattern formation that constitute self-organising systems.

\section{Reflection in self-organisation}

The previous section has endeavoured to establish that spontaneous information flow within and between an organisation and its environment, that is essential for problem solving, is one of the main attributes that leads to a self-organised system. The effectiveness of the circulation of information and its outcome is dependent on the quality of the information. The authors recommend a method of thinking and problem solving that constitutes reflection, taking into account previous experience and actions of others (and includes stigmergy). In this section, it is argued that this approach of problem solving is ideal, and in fact necessary, in a self-organised system.

It is a common concept that, when a problem arises in an organisation, one or more groups of people are formed to improve organisational performance and establish a suitable problem solving approach. Where the self-organisational approach is adopted, one of the main advantages is the flexibility to adjust to any changes within the organ- 
isation and to the environment - the real world (Checkland, 1981) - and its capacity to explore, through reflection, ongoing changes according to the system's requirement and up-to-date needs. Figure 9.4 illustrates this important process. Self-organisation can be viewed as a basis for information systems development - it is flexible; a particular approach that adjusts to specific situations but is not necessarily information systems modelling nor planning. It is a spontaneous process organised by reflection, communication and analysis of socio-technical systems.

Self-organisation can also contribute to creating a conceptual framework for non-structural, self-evolving knowledge networks. To help organisations make decisions and solve problems in a collaborative way, these knowledge networks must be present and therefore be able to reflect and share required information. This also supports information systems by allowing the adequate development of communication channels, full participation across the organisation and interaction of individuals (i.e. actors) within and between systems and the environment.

\section{Figure 9.4. The integration of reflection into self-organised processes.}

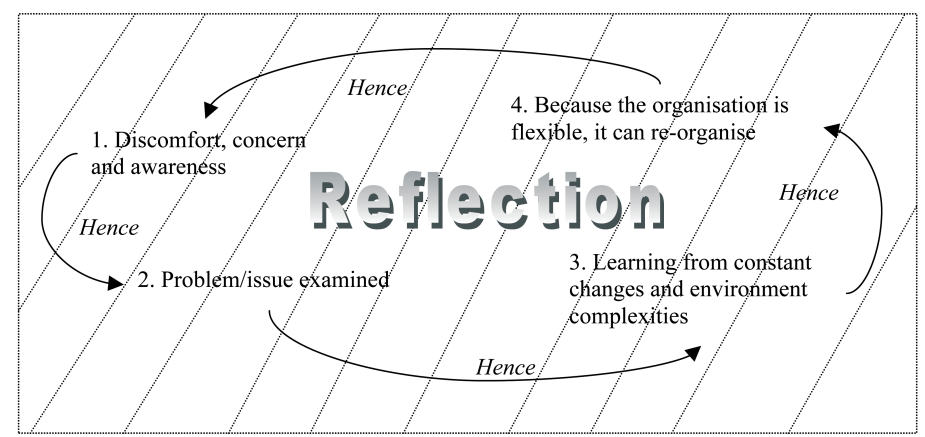

The interaction of reflection and self-organisation will be discussed by viewing the decision-making process in an organisation as being on three levels, as illustrated in Figure 9.5.

Figure 9.5. Levels of decision making in self-organised systems.

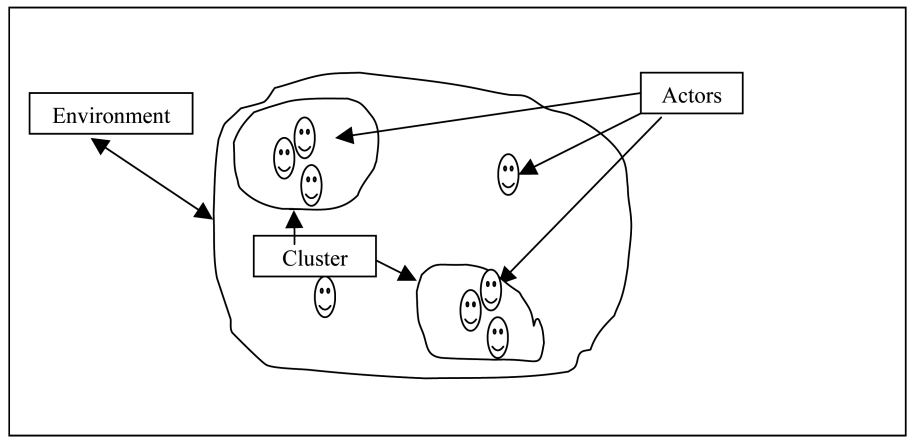

The first level consists of individuals (referred to as 'actors' in the previous section) who may be faced with an unusual or complex situation, or have an idea or conjecture about improvements to the area in which they work. The second level is composed of a grouping or cluster of individuals who have a common purpose or problem. The third 
level represents the complete system or organisation, which needs to maintain the effectiveness of its performance.

\section{Description of process}

For an organisational system to be suitable for both self-organisation and reflection, it needs to have a flat and decentralised structure, individuals need to be able to interact easily with others, and all parts of the system are necessary and sufficient for the operation of the whole but are not necessarily required to produce or replicate each other. The environment also needs to be conducive to reflective learning and cooperation between the parties. Seibert (1999) suggests the conditions that cultivate this environment are:

1. autonomy - ample freedom and discretion to structure one's work as one sees fit;

2. feedback - information on the results of one's actions - information is the raw material of reflection;

3. interactions with other people, entailing access to others - encounters with skilled and knowledgeable people; connection to others - at least one caring interpersonal relationship; and stimulation by others - encounters with people who provide new ideas and perspectives;

4. momentary solitude - periodic, brief occasions at or away from work to process new information alone.

Within an organisation that has these attributes, there will be individuals who, at any particular time, will become aware of a problem, a complex or unusual situation or perhaps an opportunity for improvement in their line of work. At this point, the individual conducts an analysis by examining the current situation, challenging their espoused theory of action, contemplating the ideal outcome and using their own experience and knowledge as well as knowledge from other sources (if it is reflection-on-action) to propose viable alternatives. The individual then tests each alternative to arrive at the optimal solution. The interaction of the individual with the information creates a spatiotemporal structure that has minimal interference from external sources. The iterative testing of alternatives within this structure creates a pattern from which cues are emitted and then perceived by other individuals in the organisation who are pursuing a similar purpose. The existence of patterns and emission of cues cause unintentional attraction between the individuals. These cues trigger an emergence of order - the creation of a cluster based on the common purpose. Within the cluster, links between individuals are strengthened as they work towards attaining a common meaning assigned to the problem or a modified purpose of this newly generated system.

This emergence of order initiates the second level. Clusters, based on various meanings are formed - each has its own purpose. Knowledge and information needs of each cluster may become part of the internal environment (stigmergy) or may form actual communication patterns (channels within the organisation) that are used to trigger the coordination and construction of activities targeting problem solving. Actors within the cluster work together, reflecting on each other's suggestions and comparing them with the ideal, often coming to a satisfactory conclusion. However, there may not be sufficient information available within the cluster, necessitating further exploration - possibly from another cluster. One important factor to consider, and that is often missed in the self-organising literature, is that cooperation is a necessary condition for the existence of a social system and therefore for the presence of clusters. Cooperation facilitates the interaction among clusters and ensures information flow so that each cluster's issues and concerns are known across the organisation. Under these conditions, clusters are better informed in a problem 
situation as their multiple interactions support further generation of information and knowledge, which is then embedded in the organisational environment.

Faced with a problem, the purpose of one particular cluster in the system will have an impact on other clusters, and vice versa (i.e. organisational networks are affected by each cluster's reflection on information and ideal). These trigger self-organisation of clusters at a different level with a much more informed image of the problem-solving ideal. Because of the cooperation among clusters, reflection from different perspectives arises, allowing the emergence of new alternatives.

At this point it is worth noting that clusters constantly accommodate in order to target best alternatives. They can therefore structure themselves, generating new patterns, stimuli, actions, and eventually new clusters. The clusters come together because of problems at an organisational level. This initiates the third level. The cooperative system carried on from the second level strengthens the availability of information from past experience, facilitating the forecasting and reflection on business trends.

At this level, an organisation is in a position to reflect upon complex environmental changes and put itself in a position where blueprints and information systems templates are no longer required. Consistent reflective processes allow the system to monitor and be aware of changes in the environment and are therefore an important asset for the organisation as they assist further development of capabilities and help ensure market position.

As pointed out earlier, decentralisation is one of the key characteristics of self-organised systems, and one that enhances reflection. A decentralised organisation does not follow a hierarchical system. In fact, they focus on a more linear structure. Here, actors are empowered to act based on their own knowledge and perceptions, and no longer have to go through extended decision channels. They start to offer solutions as soon as they are faced with a problem and they are no longer inhibited or held back waiting for instructions from superiors. There is now an internal culture for constant informationknowledge development. Being able to self-reflect indicates that the organisation has placed itself in an advantaged position by establishing a circle of learning, auto-analysis and multiple ideals for problem solving.

With each level of problem solving, there are three opportunities for reflection (Raelin, 2001):

1. Anticipatory reflection occurs prior to the relevant experience (often at the planning stage).

2. Contemporaneous reflection occurs at the moment of the relevant experience (as with Schon's reflection-in-action).

3. Retrospective reflection looks back at the relevant experience (as with reflectionon-action).

Mathiassen (2002) explains that, in systems development, the timing of reflection depends on whether the task is constructive, evolutionary or an intervention. When the task is constructive, requirements need to be taken into account to design the system required, and therefore reflection needs to be done before construction or action takes place. When dealing with evolutionary systems, the situation is already ongoing, and probably not stable, so the developer needs to reflect as the development takes place - it requires reflection-in-action. With intervention, the current situation and the problems associated with it need to be explored, alternatives suggested and implemented. This requires both reflection-in-action and reflection-on-action. 
Dewey urges that reflection take place ad infinitum. He believes one can never be certain of the 'truth' and therefore should continually search in an attempt to invalidate an idea. Each time the reflection supports the belief, it is strengthened, but reflection should never stop.

Self-organisation should also be perceived as a continual process. The systems thinking literature (e.g. Checkland, 1981) highlights the importance of flexibility within a system. Reflection supports self-organising systems' flexibility by not imposing time in the actual process of analysis and pattern formation. In a self-organising system, the flexibility of actors to adjust to the environment and anticipate problems and their solutions can only be possible by reflecting:

1. before the construction of actions takes place (i.e. prior problem recognition and awareness);

2. while the actions are taking place (i.e. during emergence of order); and

3. after actions have impacted the environment and system (i.e. learning from actions).

Actors should be able to benefit from reflecting at different times. A continuous reflecting process would guarantee the argumentation and negotiation required among actors for self-organisation to emerge. It supports the creativity needed for problem solving and helps the understanding of the underlying assumptions in complex environments. Reflection leaves actors in the position of reading the environment at any time and able to record and retrieve information and knowledge to address the ongoing requirements of the system. This type of flexibility, as described by Checkland (1981), results in the effectiveness, efficiency and efficacy of the system. Furthermore, it can support the reorganisation of systems while new patterns evolve. Success for a range of soft systems applications, issues such as complexity, variables and long-term system monitoring, are therefore addressed by the reflection rooted in self-organising systems.

\section{Conclusion}

To reiterate our argument in this paper: we suggest that a continuous reflection process is critical for successful problem solving and knowledge creation on individual, group or cluster and organisational levels in self-organised systems. An iterative reflection process allows patterns created by some individuals to attract other individuals, resulting in emergent forms that are based on a common interest. Self-organisation in human systems requires pre-existing freedom in ideas, thoughts, beliefs and actions that allows equilibrium: the system incorporates the negative and positive feedback relations that balance any fluctuations. This equilibrium results in multifunctionality, versatility and flexibility that allow the system to adapt.

A self-organising system is self-contained - it emerges as an integrated flow where actors and their collective behaviours and ideals are formed into organisational patterns and structures without any influence imposed from external sources. For this to happen, reflection within the system needs to continue but the system also needs to reflect on the state of the environment in order to be able to adapt to necessary changes. The capacity for adaptation is enhanced by the reflection process - in a self-organised system all the necessary information is available, the actor reflects on this information and is in a position to select that required for problem solution.

Within this process, communication channels support the flow of information. The ease of information flow is one of a self-organising system's characteristics and consequently it is necessary that the needed channels are available so that the most appropriate medium 
(verbal, email, phone, correspondence, voice mail, body language) can be utilised at any particular time.

To summarise, reflection is integral to the success of self-organising systems (they are intertwined). Reflection enhances:

1. the freedom of internal actors' decision-making processes (generating creativity, innovation and motivation);

2. clarification of purpose;

3. the capability of the system to adapt to the environment; and

4. expands communication channels into the environment.

\section{Future research}

Although we believe that reflection is fundamental to self-organisation, there is still much work to be done in validating this principle both in theory and in practice. Furthermore, in the information systems field, self-organisation is not clearly accepted as a problem-solving approach. We believe this approach to be feasible in the real work setting if an appropriate organisational culture is in place. It can readily be seen that reflection already occurs successfully in some organisations when addressing problems. Hence, self-organisation could easily be the next step. There will be, of course, far-ranging implications for organisations seeking to adopt this approach in terms of internal structures, information systems already in place and indeed the motivation to do so.

Acknowledgement: The authors would like to acknowledge Dr Mike Metcalfe's contribution of ideas on this topic. 